\title{
An Analysis of Evening Commute Stop-Making Behavior Using Repeated Choice Observations from a Multi-Day Survey
}

\author{
Chandra Bhat \\ Department of Civil Engineering \\ University of Texas at Austin
}

\begin{abstract}
This paper examines the number of stops made by individuals during their evening commute. The paper applies a methodological framework that relates stop-making to relevant individual, land-use, and work-related characteristics. The framework also accommodates unobserved variation in stop-making propensity across individuals in intrinsic preferences and in responsiveness to work-related attributes. The empirical analysis uses a sample of repeated choice observations from a multi-day sample of workers drawn from the 1990 San Francisco Bay area household survey. The results indicate that the proposed model provides a superior data fit relative to a model that ignores unobserved variations in stop-making propensity across individuals. The model in this paper also provides important behavioral insights which are masked by the model that disregards unobserved variations.
\end{abstract}

Keywords: Ordered-response logit, unobserved heterogeneity, random-coefficients, heteroscedasticity, maximum simulated likelihood method, transportation control measures. 


\section{Introduction}

The commute patterns of individuals have an important bearing on peak period traffic congestion. While traditional planning methods attempt to examine commute patterns primarily by analyzing the travel mode choice for the work trip, there is now an increasing body of literature that emphasizes the need to study stop-making behavior during the work commute. This is due to the growing number of nonwork stops made by individuals during the work commutes, especially during the evening work-to-home commute (see Lockwood and Demetsky, 1994; Purvis, 1994; and Davidson, 1991).

This paper focuses on modeling the number of stops made by individuals during the evening work-to-home commute. It uses a model structure that recognizes the ordinal nature of number of stops. It also explicitly accounts for variations in stop-making propensity across individuals due to a) observed (to the analyst) individual, land-use, and work-related characteristics (such as sex, income earnings and work duration), b) unobserved (to the analyst) individual characteristics (such as lifestyle/mobility preferences), and c) sensitivity differences to work-related attributes (such as differences in responsiveness to work duration). Earlier studies of trip-chaining behavior have not accommodated inter-individual variations in stop-making propensity due to the latter two effects. These two causes of variation are generally referred to as unobserved heterogeneity in econometric literature. It is now well established that ignoring unobserved heterogeneity will, in general, result in inconsistent model parameter estimates and even more severe inconsistent choice probability estimates (see Chamberlain, 1980; the reader is also referred to Hsiao, 1986 and Diggle et al., 1994 for a detailed discussion of unobserved heterogeneity bias in discrete-choice models).

In addition to accommodating unobserved heterogeneity, another related characteristic of the current modeling effort is that it recognizes the presence of day-to-day variations in stop-making behavior during the evening commute for the same individual; specifically, the estimation uses multi-day observations from individuals. Such a longitudinal (or repeated choice) sample is needed to accommodate heterogeneity due to unobserved individual characteristics since it is impossible with cross-sectional data to disentangle unobserved inter-individual differences from the effect of omitted variables that are generic across all choice occasions.

Earlier studies of trip-chaining during the evening commute have used a single day of observation (for example, see Adiv, 1983; Kondo and Kitamura, 1987; Nishii et al., 1988; Hamed 
and Mannering, 1993; Strathman et al., 1994, and Bhat, 1997). While these studies have provided valuable insights into the determinants of stop-making behavior, they implicitly assume a repetitive, non-varying, commute pattern across all days of the week and do not accommodate unobserved heterogeneity in stop-making behavior. Recently, Jou and Mahmassani (1997) have descriptively examined day-to-day variations in stop-making (along with other attributes of stop-making) during the morning and evening commutes. Their study confirms the increasing prevalence of stops during the evening commutes and also notes the day-to-day variability in evening commute stop-making. However, their work does not accommodate unobserved heterogeneity and uses a relatively restrictive model structure in the analysis.

The remainder of this paper is organized in six sections. The next section presents the model structure. Section 3 discusses the estimation technique. Section 4 describes the data source and sample used in the empirical analysis. Section 5 presents empirical results. Section 6 examines the impact of policy actions using the model. The final section provides a summary of the research findings and identifies possible extensions of the research.

\section{Model Structure}

The model structure in the current paper takes an ordered-response formulation that recognizes the ordinal nature of number of stops. The ordered-response formulation was initially proposed by McKelvey and Zavoina (1978) and has been used recently by Agyemang-Duah and Hall (1997) and Bhat (1997) to model number of stops from cross-sectional data.

Another possible model structure for number of stops is a count model (such as a Poisson or negative binomial regression). However, count models are unable to account for the ordinal nature of responses of the dependent variable and also place rather restrictive assumptions on the random error distribution (see Agyemang-Duah et al., 1995 for a discussion). Also, count models are appropriate when the dependent variable is non-categorical, but taking on only non-negative integer values (see Maddala, 1983; page 51). For the small range of stops during the evening commute (between 0 to 3 in the current sample), it is more appropriate to consider stop-making as an intrinsically discrete choice. Thus, the ordered-response structure is better suited for number of stops in the current analysis than a count model.

In the following presentation of the ordered response structure, we will use the index $k$ to represent number of stops made during the evening commute $(k=0,1,2, \ldots K)$, the index $q$ to 
represent individuals $(q=1,2, \ldots Q)$, and the index $d$ to represent workdays $\left(d=1,2, \ldots D_{q}\right)$. The number of observed workdays (i.e., evening commutes) varies across individuals with a minimum of two evening commutes to a maximum of all five evening commutes in the work week. The equation system is as follows:

$s_{q d}^{*}=\gamma_{q}^{\prime} x_{q d}+\epsilon_{q d}, s_{q d}=k$ if $\delta_{k-1}<s_{q d}^{*} \leq \delta_{k}$,

where $s_{q d}^{*}$ is the (latent) stop-making propensity of individual $q$ on day $d, x_{q d}$ is a column vector of exogenous variables, $\gamma_{q}$ is a corresponding column vector of coefficients which may vary over individuals but does not vary over days, and $\epsilon_{q d}$ is a standard logistic random term that captures the idiosyncratic effect of all omitted variables which are not individual-specific. $\epsilon_{q d}$ is assumed to be independent of $\gamma_{q}$ and $x_{q d} . s_{q d}$ is the observed number of nonwork stops made by individual $q$ on day $d$. It is characterized by the stop-making propensity $s_{q d}^{*}$ and the threshold bounds (the $\delta$ 's) in the usual ordered-response fashion $\left(\delta_{-1}=-\infty\right.$ and $\left.\delta_{K}=+\infty\right)$.

Let us partition the vector $\gamma_{q}=\left[\eta_{q}, \beta_{q}^{\prime}\right]^{\prime}$ and correspondingly the vector $x_{q d}=\left[1, y_{q d}^{\prime}\right]^{\prime}$. $\eta_{q}$ is an individual-specific scalar term that affects stop-making propensity. $\beta_{q}$ is a column vector of coefficients on an observed vector $y_{q d}$ of work-related and (possibly) other non-individual specific variables.

Let the individual-specific term be written as a linear function of observed individual characteristics: $\eta_{q}=\lambda^{\prime} z_{q}$, where $z_{q}$ is a column vector of observed individual characteristics and $\lambda$ is a corresponding column vector fixed across all individuals. Also, let $\beta_{q}=\beta$ for all individuals $q$. This specification corresponds to the standard ordered-response logit (ORL) formulation which ignores inter-individual differences due to unobserved individual characteristics and due to variations in sensitivity to work-related/other variables.

An alternative and more general specification is to specify the individual-specific term as the sum of an unobserved component $\alpha_{q}$ and a linear function of observed individual variables: $\eta_{q}=\alpha_{q}+\lambda^{\prime} z_{q}$. Let $\alpha_{q}$ have a normal distribution across individuals with a mean of zero (the restriction on the mean is an innocuous one because of the inclusion of the thresholds). The variance of $\alpha_{q}$ captures intercept (or intrinsic) unobserved heterogeneity in stop-making propensity across individuals. One may assume this variance to be fixed across individuals or permit the variance to differ across individual groups. The latter formulation is a generalization of the former 
and may be more appropriate. For example, there might be more variance in stop-making propensity within the group of individuals who are single than the group of individuals who live with others (individuals living alone have fewer responsibilities and hence can exercise greater choice in stop-making). Alternatively, the intercept unobserved heterogeneity may be higher among women than among men since men might make no stops more consistently than do women. In this paper, we allow for such differences in intercept unobserved heterogeneity by specifying the variance of $\alpha_{q}$ to be a function of individual attributes. That is, $\alpha_{q} \sim N\left[0, \sigma_{q}^{2}\right]$ with $\sigma_{q}=$ $\exp \left(\omega+\mu^{\prime} w_{q}\right)$, where $w_{q}$ is a vector of individual attributes. The exponential functional form is used in the standard error specification to ensure its non-negativity [Greene (1997, p. 889), McMillen (1995), and Swait and Adamowicz (1996) also use an exponential form for accommodating heteroscedasticity in discrete choice models]. In addition to intercept unobserved heterogeneity and heteroscedasticity in the intercept unobserved heterogeneity, we also accommodate variations in sensitivity to work-related/other attributes by allowing the elements of $\beta_{q}$ to be randomly (normally) distributed across individuals (the distributions of the elements are assumed to be independent). That is, $\beta_{q j} \sim N\left(\beta_{j}, \Omega_{j}^{2}\right)$, where $j$ is an index for the elements in $\beta_{q}$.

With the specifications discussed above, Equation (1) may be written as:

$$
\begin{aligned}
& s_{q d}^{*}=\alpha_{q}+\lambda^{\prime} z_{q}+\sum_{j} \beta_{q j} y_{q d j}+\epsilon_{q d}, \alpha_{q} \sim N\left(0,\left[\exp \left(\omega+\mu^{\prime} w_{q}\right)\right]^{2}\right), \beta_{q j} \sim N\left(\beta_{j}, \Omega_{j}^{2}\right), \\
& s_{q d}=k \text { if } \delta_{k-1}<s_{q d}^{*} \leq \delta_{k} .
\end{aligned}
$$

The above model form corresponds to a random-coefficients heteroscedastic ordered response logit (RCHORL) formulation. The reader will note that the subscript $d$ in the above equation disappears with cross-sectional data and one cannot separate out the individual-specific deviation term $\alpha_{q}$ from the effect of omitted variables that are not individual-specific $\epsilon_{q d}$; that is, with cross-sectional data, we cannot accommodate unobserved heterogeneity in the intercept.

Conditional on the $\alpha_{q}$ and $\beta_{q}$ terms $(j=1,2, \ldots J)$, we get the familiar ordered-response logit form for the choice probability of individual $q$ making $k$ number of stops on day $d$ ( $L$ represents the logistic distribution function below): 
$P_{q d k} \mid\left(\alpha_{q}, \beta_{q 1}, \beta_{q 2}, \ldots, \beta_{q J}\right)$

$=L\left(\delta_{k}-\alpha_{q}-\lambda^{\prime} z_{q}-\sum_{j} \beta_{q j} y_{q d j}\right)-L\left(\delta_{k-1}-\alpha_{q}-\lambda^{\prime} z_{q}-\sum_{j} \beta_{q j} y_{q d j}\right)$

The unconditional probability of choosing number of stops $k$ for a randomly selected individual with observed vectors $z_{q}, y_{q d}$, and $w_{q}$ can now be obtained by integrating the conditional choice probabilities in Equation (3) with respect to the assumed random (and independent) normal distributions for the $(J+1)$ random variables $\alpha_{q}, \beta_{q 1}, \beta_{q 2}, \ldots, \beta_{q J}$. The resulting expression has the following form:

$$
\begin{aligned}
P_{q d k}= & \int_{-\infty}^{+\infty} \int_{-\infty}^{+\infty} \cdots \int_{-\infty}^{+\infty}\left[L\left(\delta_{k}-\alpha_{q}-\lambda^{\prime} z_{q}-\sum_{j} \beta_{q j} y_{q d j}\right)-L\left(\delta_{k-1}-\alpha_{q}-\lambda^{\prime} z_{q}\right.\right. \\
& \left.\left.-\sum_{j} \beta_{q j} y_{q d j}\right)\right] \mathrm{d} F\left(\alpha_{q}\right) \mathrm{d} F\left(\beta_{q 1}\right) \mathrm{d} F\left(\beta_{q 2}\right) \ldots \mathrm{d} F\left(\beta_{q J}\right) .
\end{aligned}
$$

\section{Model Estimation}

The parameters to be estimated in the random-coefficients heteroscedastic ordered response logit (RCHORL) model of Equation (2) include the vector $\zeta=\left(\omega, \mu, \lambda^{\prime}\right)^{\prime}$ and the vector $\xi_{j}=\left(\beta_{j}, \Omega_{j}\right)$ for $j=1,2, \ldots$ J. Let $\tau=\left(\zeta^{\prime}, \xi_{1}^{\prime}, \xi_{2}^{\prime}, \ldots, \xi_{J}^{\prime}\right)^{\prime}$ represent the full set of parameters to be estimated. To develop the likelihood function, we need the probability of each sample individual's sequence of observed number of stops choice. Conditional on $\alpha_{q}, \beta_{q 1}, \ldots, \beta_{q J}$, the likelihood function for individual $q$ 's observed sequence of choices is:

$L_{q} \mid\left(\alpha, \beta_{q 1}, \ldots, \beta_{q K}\right)=\prod_{d=1}^{D_{q}} \prod_{k=1}^{K}\left[P_{q d k} \mid\left(\alpha_{q}, \beta_{q 1}, \ldots, \beta_{q K}\right)\right]^{M_{q d k}}$, where

$M_{q d k}=\left\{\begin{array}{l}1 \text { if the } q \text { th individual makes } k \text { stops on day } d \\ 0 \text { otherwise }\end{array}\right.$

The unconditional likelihood function of the choice sequence is:

$$
L_{q}(\tau)=\int_{-\infty}^{+\infty} \int_{-\infty}^{+\infty} \cdots \int_{-\infty}^{+\infty}\left\{L_{q} \mid\left(\alpha_{q}, \beta_{q 1}, \ldots, \beta_{q J}\right)\right\} \mathrm{d} F\left(\alpha_{q}\right) \mathrm{d} F\left(\beta_{q 1}\right) \ldots \mathrm{d} F\left(\beta_{q J}\right) .
$$


Now define $r_{q}$ and $u_{q j}(q=1, \ldots, Q, j=1, \ldots, J)$ as standard-normal variates so that $r_{q}=$ $\alpha_{q} /\left[\exp \left(\omega+\mu^{\prime} w_{q}\right)\right]$ and $u_{q j}=\beta_{q j} / \Omega_{j}$. Then, using Equations (3) and (5), the unconditional likelihood function of Equation (6) may be written for a given value of the parameter vector $\tau$ as:

$$
\begin{aligned}
L_{q}(\tau)= & \int_{-\infty}^{+\infty} \int_{-\infty}^{+\infty} \cdots \int_{-\infty}^{+\infty}\left[\prod _ { d = 1 } ^ { D _ { q } } \left\{\prod _ { k = 1 } ^ { K } \left[L\left(\delta_{k}-\left[\exp \left(\omega+\mu^{\prime} w_{q}\right)\right] r_{q}-\lambda^{\prime} z_{q}-\sum_{j} \Omega_{j} u_{q j} y_{q d j}\right)\right.\right.\right. \\
& \left.\left.\left.-L\left(\delta_{k-1}-\left[\exp \left(\omega+\mu^{\prime} w_{q}\right)\right] r_{q}-\lambda^{\prime} z_{q}-\sum_{j} \Omega_{j} u_{q j} y_{q d j}\right)\right]^{M_{q d k}}\right\}\right] \\
& d \Phi\left(r_{q}\right) d \Phi\left(u_{q 1}\right) d \Phi\left(u_{q 2}\right) \ldots d \Phi\left(u_{q J}\right)
\end{aligned}
$$

where $\Phi($.$) represents the standard normal distribution function. The log-likelihood function is$ $\mathcal{L}(\tau)=\sum_{q} \ln L_{q}(\tau)$.

The log-likelihood function involves the evaluation of a $(J+1)$-dimensional integral $(J$ is the number of variables with random response coefficients). Conventional quadrature techniques cannot compute the integrals with sufficient precision and speed for estimation via maximum likelihood when the dimensionality of the integration is greater than two (in the empirical analysis in Section 5, the dimensionality of the integration is five).

In the current study, we apply Monte Carlo simulation techniques to approximate the integrals in Equation (7) and maximize the resulting simulated log-likelihood function. The simulation technique computes the integrand in Equation (7) at randomly chosen values for each $r_{q}$ and $u_{q j}$. Specifically, we draw a particular realization of $r_{q}$ and $u_{q j}(j=1,2, \ldots J)$ by generating a vector of $(J+l)$ standard normal random numbers for each individual $q$ and subsequently compute the integrand in Equation 7 for a given value of the parameter vector $\tau$. We then repeat this process $N$ times for each individual for the given value of the parameter vector $\tau$. Let $\tilde{L}_{q}^{n}(\tau)$ be the realization of the individual likelihood function in the $n^{\text {th }}$ draw $(n=1,2, \ldots N)$. The individual likelihood function is then approximated by averaging over the $\tilde{L}_{q}^{n}(\tau)$ values:

$\tilde{L}_{q}(\tau)=\frac{1}{N} \sum_{n=1}^{N} \tilde{L}_{q}^{n}(\tau)$

where $\tilde{L}_{q}(\tau)$ is the simulated likelihood function for the $q^{\text {th }}$ individual's sequence of choices given the parameter vector $\tau . \tilde{L}_{q}(\tau)$ is an unbiased estimator of the actual likelihood function 
$L_{q}(\tau)$. Its variance decreases as $N$ increases. It also has the appealing properties of being smooth (i.e., twice differentiable) and being strictly positive for any realization of the finite $N$ draws.

The simulated log-likelihood function is constructed as:

$S \mathcal{L}(\tau)=\sum_{q=1}^{Q} \log \left[\tilde{L}_{q}(\tau)\right]$

The parameter vector $\tau$ is estimated as the vector value that maximizes the above simulated function. Under rather weak regularity conditions, the maximum simulated log-likelihood (MSL) estimator is consistent, asymptotically efficient, and asymptotically normal (see Hajivassiliou and Ruud, 1994; Lee, 1992). In the current paper, we use 500 repetitions for accurate simulations of the individual log-likelihood functions and to reduce simulation variance of the MSL estimator [the simulation approach discussed above has been used earlier by Revelt and Train (1997), Train (1997), and Bhat (1998a) in the context of a multinomial logit model].

All estimations and computations were carried out using the GAUSS programming language on a personal computer. Gradients of the simulated log-likelihood function with respect to the parameters were coded.

\section{Data Source and Sample Used}

The data source for the analysis is the San Francisco Bay Area Household Travel Survey conducted by the Metropolitan Transportation Commission (MTC) in the Spring and Fall of 1990. This survey collected a multiple-weekday (either 3-day or 5-day) travel diary for some households, and it is this multi-day sample that is used here. In addition to the travel diary, the survey also collected individual and household socio-demographic information. The survey contacted about 1500 Bay Area households by telephone using a random selection process for telephone numbers. This was followed by the mailing of travel diary cards to the households, and retrieval of travel diary data by follow-up telephone calls (see White and Company, Inc., 1991 for details of survey sampling and administration procedures).

The sample for the current analysis comprises 1669 person-days in which an evening commute was undertaken. The 1669 person-days corresponds to 533 individuals: 140 of these individuals had 2 days of useable information, 259 had 3 days, 58 had 4 days, and 76 had all 5 days of useable information (only those individuals who had at least 2 days of useable commute 
information were selected into the sample; about $18 \%$ of employed individuals in the multi-day survey sample had only 1 day of commute information and these individuals were removed from the sample used in analysis).

Activities pursued for all purposes except for the sole purpose of changing travel modes (such as changing from transit to drive alone at a transit station) were considered as a stop in the evening commute. The distribution of the number of evening commute stops in the person-day sample was as follows: $0(67.6 \%), 1(23.4 \%), 2(6.4 \%)$ and greater than or equal to $3(2.6 \%)$. These statistics indicate that almost a third of all commuters make one or more stops during the evening commute on a weekday. It is also interesting to note the rather high number of multiple stops: among those who make any evening commute stops, almost 38.5\% make more than one stop.

Table 1 presents the distribution of evening commute stops at an individual level. The table indicates that only $37.7 \%$ of individuals made no stops on all days of their observed evening commutes. This shows that evening stop-making is much more prevalent when viewed over a period of multiple days than on any given day. The last two rows of the table show that only $43.3 \%$ of individuals make the same number of stops across all days, while the remaining $56.7 \%$ of individuals do not have a consistent stop-making pattern. This is indicative of the substantial day-to-day variation in evening stop-making and emphasizes the need to study evening stop-making behavior from a multi-day sample rather than a single-day sample.

Table 1: Multi-day Evening Commute Stop-Making Pattern of Individuals

\begin{tabular}{||l|c|c||}
\hline \multicolumn{1}{|c|}{ Stop-making pattern } & $\begin{array}{c}\text { Number of } \\
\text { individuals }\end{array}$ & $\begin{array}{c}\text { Percentage of } \\
\text { individuals }\end{array}$ \\
\hline \hline Zero stops across all days & 201 & 37.7 \\
One stop across all days & 27 & 5.0 \\
Two stops across all days & 2 & 0.4 \\
Three stops across all days & 1 & 0.2 \\
\hline Same number of stops across all days & 231 & 43.3 \\
Different number of stops across days & 302 & 56.7 \\
\hline
\end{tabular}

\section{Empirical Analysis}

\subsection{Variable Specification}

Three sets of variables were considered to explain evening stop-making propensity in this study. They were a) individual and household socio-demographics, b) retail employment densities at the home and work places, and c) work-related attributes. 
Among individual and household socio-demographics, the sex of the individual, presence of children less than 5 years in the household, family structure variables (whether single individual or couple family households), and ownership indicator of the household had a statistically significant impact on evening stop-making propensity. Several other variables such as age of individual, race of individual (whether caucasian or not), presence of children greater than 5 years, number of other employed adults, number of unemployed adults, and household income did not significantly impact stop-making propensity.

The retail employment densities at the home/work places were computed as the total retail employment at the home/work traffic zones divided by the total acreage of the home/work zones. These variables were introduced to proxy the effect of density of stop-making opportunities at the home/work ends. A high work-end retail density may lead to more stop-making because of increased activity opportunities if individuals pursue stops near their work place. A high home-end retail density may increase commute stop-making if individuals pursue activities closer to their home. On the other hand, it may decrease commute stops if individuals decide to pursue activities after arriving home first.

Four variables were introduced within the set of work-related attributes. These included work duration, travel time to work, an indicator for departure from work between 4 and 7 pm, and another indicator for departure from work after $7 \mathrm{pm}$. Work duration was computed as the time difference between departure from work in the evening and arrival at work in the morning. The travel time to work corresponds to the direct work-to-home time (without any stop-making) and is obtained from network travel impedance data (a detailed description of the procedures and assumptions employed in arriving at the impedance data is beyond the scope of the current paper, but is available in Purvis, 1996). Departure before $4 \mathrm{pm}$ is used as the base category in specifying the departure time variables. In the group of work-related attributes, we also considered a fifth variable: travel mode to work. However, this variable did not have any significant impact on stop-making propensity possibly because a very high fraction of individuals in the sample used the car mode with little market penetration of other modes.

A point to note here about the work-related variables. It is possible that these variables are endogenous to evening commute stop-making, especially if individuals have substantial flexibility in their work schedules. The assumption in this study (as in almost all previous studies of evening commute stops, for example, see Hamed and Mannering, 1993, Jou and Mahmassani, 1997, and 
Bhat, 1997) is that work schedules are pre-determined, and that the evening commute stops decision is conditional on the work schedule. In general, it is quite reasonable to assume that work duration on each day of the week is pre-determined (though it may differ across days of the week based on employment arrangements). While there may be some flexibility in departure time from work based on the number of evening commute stops an individual would like to make, the "bandwidth" of this flexibility is likely to be smaller than the broad time periods adopted for departure time in this study. Further, it should be recognized that work schedule change-related transportation control measures implicitly consider work schedules to be pre-determined. Assessing the impact of such TCMs can therefore be pursued only by assuming work schedules to be pre-determined. Nonetheless, a careful examination and modeling of work schedule along with evening commute stop-making to accommodate jointness in these two choices is an important area for further research.

Table 2 provides the descriptive sample statistics of all the exogenous variables used to explain evening commute stop-making behavior.

Table 2: Descriptive Sample Statistics of Exogenous Variables

\begin{tabular}{||l|c|c|c|c||}
\hline \hline Variable & Mean & Std. Dev. & Min. & Max. \\
\hline \hline Individual/household socio-demographics & & & & \\
Female & 0.42 & 0.49 & 0.00 & 1.00 \\
Presence of children < 5 years & 0.13 & 0.34 & 0.00 & 1.00 \\
Single person family & 0.17 & 0.37 & 0.00 & 1.00 \\
Couple family & 0.26 & 0.44 & 0.00 & 1.00 \\
Own home $^{\text {Retail employment densities }}{ }^{\mathrm{a}}$ & 0.63 & 0.48 & 0.00 & 1.00 \\
Work end x 10 $^{-3}$ & & & & \\
Home end x 10 & 1.04 & 1.21 & 0.00 & 8.19 \\
\hline Work-related attributes & 0.47 & 0.50 & 0.00 & 3.59 \\
Work duration (minutes) x 10-2 $^{-3}$ & & & & \\
Commute travel time (minutes) x 10-1 & 5.28 & 1.06 & 1.05 & 9.94 \\
Departure between 4-7 pm & 2.52 & 1.63 & 0.18 & 10.50 \\
Departure after 7 pm & 0.66 & 0.47 & 0.00 & 1.00 \\
\hline
\end{tabular}

${ }^{a}$ The retail employment density for a zone is computed as the total retail employment in the zone divided by the number acreage of the zone.

\section{Empirical Results}

The parameter estimation results for the standard ordered response logit (ORL) model and the random coefficients heteroscedastic ordered response logit (RCHORL) model are provided in 
Table 3. Prima facie, parameters estimates are similar in sign in the two models. The effect of individual and household socio-demographics indicates that women are more likely to make evening commute stops than men. This is consistent with the finding of many earlier studies (see, for example, Bianco and Lawson, 1996; Mensah, 1995), possibly reflecting the continuing trend of women to shoulder a major part of household maintenance responsibilities. Individuals with small children (less than five years of age) are likely to pursue more evening commute stops than individuals with no small children. Individuals who live alone or in a couple family household have a higher stop-making propensity than individuals in other households. This may reflect the lesser familial responsibilities of individuals in single and couple family households compared to other household types. Finally, the results also indicate a higher stop-making propensity for individuals who own their homes relative to renters. The effect of this variable is not statistically significant in the RCHORL model.

The retail employment density variables indicate a higher propensity to make stops if the work place is located in a zone with high retail density. This is quite reasonable, since it suggests more stop-making as the density of opportunities for stop-making around the work place increases. A similar, but statistically insignificant effect is observed for the effect of retail density at the home end.

The sensitivity to work-related attributes is assumed to be fixed across individuals in the ORL model. The ORL model indicates that a higher work duration reduces evening commute stop-making propensity. This may be a consequence of constraints on the amount of time available for post-work activity participation. The effect of commute travel time appears to have a positive effect on stop-making propensity. The departure time variables imply greater stop-making propensity for individuals who leave work before $4 \mathrm{pm}$, possibly due to greater time availability in the evening.

The RCHORL model estimates more parameters than the ORL model since it accommodates variations in sensitivity to work-related attributes and intercept unobserved heterogeneity. The mean coefficients of the work-related variables in the RCHORL model are, in general, higher in magnitude than the ORL model. The standard deviations of the parameter estimates on the work-related attributes (except for the "departure after $7 \mathrm{pm}$ " variable) are statistically significant and fairly high relative to the mean in the RCHORL model. Thus, there is heterogeneity in sensitivity to work-related attributes. 
Table 3: Estimation Results for Stop-Making Propensity

\begin{tabular}{|c|c|c|c|c|c|}
\hline \multirow{2}{*}{\multicolumn{2}{|c|}{ Variable $^{\mathrm{a}}$}} & \multicolumn{2}{|c|}{$\begin{array}{c}\text { Standard ordered response } \\
\text { logit (ORL) }\end{array}$} & \multicolumn{2}{|c|}{$\begin{array}{l}\text { Random coeffs. heter. ordered } \\
\text { response logit (RCHORL) }\end{array}$} \\
\hline & & Parameter & t-stat. & Parameter & t-stat. \\
\hline \multicolumn{6}{|c|}{ Individual/household socio-demographics } \\
\hline \multicolumn{2}{|l|}{ Female } & 0.255 & 2.35 & 0.222 & 1.64 \\
\hline \multicolumn{2}{|l|}{ Presence of children $<5$ years } & 0.385 & 2.24 & 0.426 & 1.50 \\
\hline \multicolumn{2}{|l|}{ Single person family } & 0.955 & 6.39 & 1.056 & 2.67 \\
\hline \multicolumn{2}{|l|}{ Couple family } & 0.647 & 4.88 & 0.735 & 3.10 \\
\hline \multicolumn{2}{|l|}{ Own home } & 0.256 & 2.17 & 0.273 & 1.38 \\
\hline \multicolumn{6}{|c|}{ Logarithm of Retail employment densities } \\
\hline \multicolumn{2}{|l|}{ Work end } & 0.133 & 2.49 & 0.159 & 2.01 \\
\hline \multicolumn{2}{|l|}{ Home end } & 0.072 & 1.44 & 0.057 & 0.72 \\
\hline \multicolumn{6}{|l|}{ Work-related attributes } \\
\hline \multirow[t]{2}{*}{ Work duration $\times 10^{-2}$} & - Mean & -0.209 & -3.64 & -0.335 & -3.87 \\
\hline & - Std. dev. & - & - & 0.100 & 2.22 \\
\hline \multirow[t]{2}{*}{ Commute time $\times 10^{-1}$} & - Mean & 0.084 & 2.51 & 0.098 & 1.67 \\
\hline & - Std. dev. & - & - & 0.156 & 2.08 \\
\hline \multirow{2}{*}{ Departure between 4-7 pm } & - Mean & -0.689 & -5.29 & -0.971 & -5.30 \\
\hline & - Std. dev. & - & - & 0.263 & 3.15 \\
\hline \multirow[t]{2}{*}{ Departure after $7 \mathrm{pm}$} & - Mean & -0.700 & -3.05 & -1.027 & -2.53 \\
\hline & - Std. dev. & - & - & 0.016 & 0.43 \\
\hline \multicolumn{6}{|c|}{ Intercept unobserved heterogeneity } \\
\hline \multicolumn{2}{|l|}{ Constant } & - & - & -0.111 & - \\
\hline \multicolumn{2}{|l|}{ Female } & - & - & 0.192 & 2.13 \\
\hline \multicolumn{2}{|l|}{ Single person household } & - & - & 0.313 & 3.80 \\
\hline \multicolumn{2}{|l|}{ Log-likelihood at convergence $^{b}$} & \multicolumn{2}{|c|}{-1388.03} & \multicolumn{2}{|c|}{-1334.81} \\
\hline
\end{tabular}

${ }^{a}$ The threshold values and their standard errors (in parenthesis) for the ORL model are $0.6248(0.40), 2.308(0.39)$ and $3.666(0.41)$. The corresponding values for the RCHORL model are $0.036(0.62), 2.096(0.62)$ and $3.642(0.67)$.

${ }^{\mathrm{b}}$ The log-likelihood value at convergence for several other models are as follows: a) with threshold values only (i.e., market share model): $-1460.65, \mathrm{~b}$ ) with threshold values and independent variables only: $-1388.03, \mathrm{c}$ ) with threshold values, independent variables and homoscedastic intercept unobserved heterogeneity: -1360.05 , d) with heteroscedasticity in the intercept heterogeneity added to specification c: -1342.51 , e) with heterogeneity in responsiveness to work-related attributes added to specification c: -1343.62 
The estimates of the mean and standard deviation of the work duration coefficient indicate that stop-making propensity decreases with work duration for almost all individuals (less than $0.05 \%$ of the population have a positive coefficient, as implied by the normal distribution of the work duration coefficient). ${ }^{1}$

The estimates for the commute travel time indicate that a larger commute travel time increases stop-making propensity for about three-fourths of the population, while it decreases stop-making propensity for the remaining one-fourth of the population. It is possible that a longer commute time is associated with more density of opportunities for activity participation for some individuals while it imposes temporal constraints on others, leading to the different directions in effect. The RCHORL model indicates a marginally significant positive mean parameter on commute travel time and a statistically significant standard deviation parameter, emphasizing the variation in responsiveness to commute travel time. The ORL model, on the other hand, estimates a small (but statistically significant) positive response across all individuals. It masks the substantial variation and different directionality of effects in the response across individuals.

The estimates for the mean coefficient of the two departure time variables in the RCHORL model are about the same and negative indicating that, on average, individuals make fewer trips if they leave work after $4 \mathrm{pm}$ than before $4 \mathrm{pm}$. However, the extent of variation across individuals is quite different between the two departure time variables. There is significant and substantial variation in stop-making propensity across individuals who leave work between 4 to $7 \mathrm{pm}$, while there is a high consistency in the reduced stop-making propensity among individuals who leave work after $7 \mathrm{pm}$. The latter result may be a reflection of the strong and uniform effect of constraints on time availability for individuals who leave work after $7 \mathrm{pm}$. The normal distribution assumption on the parameters in the RCHORL model necessarily implies a positive response parameter for the departure time variables for some share of the population. However, the mean and standard deviation estimates indicate that this share is zero for all practical purposes; that is, it is almost universally true that individuals will make more stops if they leave work before 4 pm than after.

In addition to allowing variations in responsiveness to work-related attributes, the RCHORL model also accommodates intrinsic differences in stop-making propensity across

\footnotetext{
${ }^{1}$ The normal distribution assumption implies that some share of the population will have a positive coefficient for work duration. Alternatively, one can use a log-normal distribution on the coefficient of work duration to impose a negative coefficient for all individuals. When we did so, the log-likelihood of the resulting model was slightly lower than the one with a normally distributed coefficient.
} 
individuals (i.e., intercept unobserved heterogeneity). To allow differences in the amount of heterogeneity across individual groups, we use an exponential form to relate individual attributes to the standard deviation characterizing the intercept heterogeneity (see section 2). The only two variables found to significantly affect this standard deviation were the female sex indicator and the indicator for single person households, as shown toward the bottom of Table 3 . The table does not indicate a t-statistic for the constant under intercept unobserved heterogeneity because the only logical statistical test for the value of this parameter is with negative infinity, corresponding to the absence of intercept unobserved heterogeneity. However, when individual attributes are not introduced in the unobserved heterogeneity term, one can directly estimate the fixed variance (without using an exponential functional form) and compare this variance estimate with zero to test for presence of intercept unobserved heterogeneity (maintaining homoscedasticity of the variance parameter). The resulting variance parameter is 0.9758 and the t-statistic with respect to zero is 6.20. This indicates the presence of statistically significant intercept unobserved heterogeneity. Of course, the RCHORL model in Table 3 is more general and allows heteroscedasticity in the intercept unobserved heterogeneity. The results indicate more variability in stop-making across females (relative to males) and across single individual households (relative to other family types).

\section{Application of the Model}

The model estimated in this paper can be used to determine the change in the number of nonwork stops during the evening commute due to changes in socio-demographic characteristics over time or due to policy actions that alter the work schedule of individuals. In this paper, we demonstrate the application of the model by studying the effect of two work schedule-related transportation control measures (TCMs). The two TCMs are work staggering and an increase in daily work duration due to a compressed work week policy. In examining the impact of these TCMs, it is critical to assess their effect on nonwork stops. This is the focus of the current section.

The work staggering policy is "implemented" by randomly selecting $25 \%$ of observations in the sample with a departure from work between $4 \mathrm{pm}$ and $6 \mathrm{pm}$ and subtracting 120 minutes from the departure time. The result is that the work departure time for these person-days is staggered to before $4 \mathrm{pm}$. The original departure time distribution from work in the sample is as follows: 403 (24\%) leave before 4 pm, 1098 (66\%) leave between 4 and 7 pm, and 168 (10\%) 
leave after $7 \mathrm{pm}$. After "implementing" the work staggering policy, the departure time distribution is altered: 625 (37\%) leave before 4 pm, 876 (53\%) leave work between 4 and 7 pm, and 168 $(10 \%)$ leave work after $7 \mathrm{pm}$.

The work week compression policy is realized by increasing the daily work duration of a subset of individuals by $25 \%$ (this results in a 4 day work week with the same number of total weekly work duration as the original 5 day work week). The subset (for which the work duration is increased) comprises individuals who depart work between 4 and $6 \mathrm{pm}$ and work less than or equal to 8 hours on all their observed commute days. The work duration on each (and all) days of such individuals is increased by 25\%. There are 107 individuals (311 person-days) whose work durations are increased as a result. The average work duration before and after the increase is 528 minutes and 548 minutes, respectively. We assume that the increase in work duration is equally split between an earlier arrival to work in the morning and a later departure from work in the evening. Thus, even after the increase in work duration, the latest work departure for individuals in the subset is still before $7 \mathrm{pm}$ on all their commute days.

An important note before proceeding to analyze the impact of work-related policies using the model in this paper. The model assumes that the only possible impact of work-schedule policies is on changing the number of evening commute stops. In reality, the behavioral response to work-related policies can be much more complex and may include a) reallocation of activities among individuals in a household (Jones et al., 1993), b) changes in activity duration and activity location of evening commute stops (see Bhat, 1998b), c) substitution in activities between the evening commute and after arriving back home from work (Bhat and Singh, 1998), d) substitution of out-of-home activities with in-home activities (Kitamura et al., 1996), and e) shifting of activities to non-work days. The current model is unable to accommodate such complex responses because it adopts a rather myopic perspective by ignoring the multiple dimensions characterizing activity behavior. On the other hand, while some of the studies listed above do model several activity attributes jointly, they do not accommodate unobserved heterogeneity across individuals or the intra-individual day-to-day variations in activity behavior. Ideally, it would be useful to integrate the multi-dimensional nature of some of the earlier studies with the longitudinal perspective of the current study. This is an area for future research.

The impact of the policy actions in the context of the current model is evaluated by modifying exogenous variables to reflect a change, computing revised expected aggregate values 
for number of trips in each stop category, and then obtaining a percentage change from the baseline estimates. Table 4 provides the results estimated by the ordered-response logit (ORL) model and the random coefficients heteroscedastic ordered-response logit (RCHORL) model.

Table 4: Impact of Policy Actions on Number of Evening Commute Stops

\begin{tabular}{||l||c||c|c|c|c|c||}
\hline \multirow{2}{*}{ Policy Scenario } & \multirow{2}{*}{ Model $^{\mathrm{a}}$} & \multicolumn{4}{|c|}{ Percentage aggregate change in stops during the evening commute } \\
\cline { 2 - 7 } & & 0 stops & 1 stop & 2 stops & 3 stops & Net effect \\
\hline \hline \multirow{2}{*}{ Work staggering } & ORL & -2.920 & 5.098 & 8.151 & 9.226 & 6.714 \\
\cline { 2 - 7 } & RCHORL & -4.076 & 6.721 & 11.142 & 13.107 & 9.239 \\
\hline $\begin{array}{l}\text { Work-week } \\
\text { compression } \\
\text { (increase in daily } \\
\text { work duration) }\end{array}$ & ORL & 1.333 & -2.153 & -4.080 & -4.764 & -3.174 \\
\cline { 2 - 7 } & RCHORL & 2.275 & -3.424 & -6.900 & -8.386 & -5.392 \\
\hline \hline
\end{tabular}

${ }^{a}$ ORL refers to the standard ordered-response logit model; RCHORL refers to the random-coefficients heteroscedastic ordered-response logit model.

In response to the work staggering policy, the ORL and RCHORL models indicate a reduction in the number of zero stops and progressively higher percentage increases in one, two, and $\$$ three stops. This is a consequence of the overall positive effect of an early departure from work on evening commute stop-making propensity. An important observation from the results is that while staggering work departure to an earlier time of day may reduce peak-period work-to-home trips, it also generates additional trips due to increased commute stop-making. Thus, there is some tempering of the positive benefit of a work staggering policy. Between the ORL and RCHORL model, the ORL model predicts a smaller percentage decrease in zero stops and smaller percentage increases in the one, two, and \$ three stop categories.

In response to a work-week compression policy (i.e., an increase in daily work duration for a sub-sample), both the ORL and RCHORL model predict an increase in the number of evening commutes with zero stops and a progressive percentage decrease in higher number of stops. This is a result of the overall negative effect of work duration on stop-making. The ORL model, however, projects a lower percentage increase in zero stops and a lower percentage decrease in higher number of stops. 
The net percentage change in evening commute stops can be computed as:

Net $\%$ change in evening commute stops $=\sum_{k=0}^{K}\left(\frac{k h_{k}}{\sum_{k} k h_{k}}\right) \theta_{k}$

where $h_{k}$ is the expected number of individuals who have an evening commute characterized by $k$ stops and $\theta_{k}$ is the percentage aggregate change in each stop category. This overall effect on number of stops is shown in the final column of Table 2. The ORL model underestimates the increase in evening commute stops due to a work staggering policy by as much as $28 \%$. It also underestimates the decrease in commute stops due to a work-week compression policy by about $41 \%$. Since each non-work stop contributes an additional trip in the evening period, the incorrect predictions from the ORL model can lead to the mis-guided implementation of transportation control measures.

\section{Summary and Conclusions}

This paper uses an ordered-response logit structure for repeated choice data that accommodates unobserved heterogeneity across individuals in the intercept preference term as well as in the responsiveness to relevant exogenous variables. The model also allows the intercept heterogeneity to be heteroscedastic across individual groups. The resulting Random Coefficients Heteroscedastic Ordered Response Logit (RCHORL) model is estimated using a maximum simulated likelihood method.

The empirical analysis uses the 1991 Bay area multi-day household survey to examine the stop-making behavior of commuters during their evening work-to-home journey. The results indicate the strong effects of individual and household socio-demographics, the retail employment density at the work place, and work schedule characteristics. The analysis also shows the significant presence of intercept unobserved heterogeneity in stop-making propensity across individuals, heteroscedasticity in the intercept unobserved heterogeneity, and heterogeneity in responsiveness to work-related attributes. Accommodating these sources of unobserved heterogeneity leads to a statistically superior data fit and provides behavioral insights that cannot be obtained otherwise.

The paper applies the standard ordered response (ORL) model and the RCHORL model proposed in this paper to evaluate the effect of a work staggering policy and a work week 
compression policy. The ORL model underestimates the increase in evening commute stops due to a work staggering policy and also underestimates the decrease in commute stops due to the work week compression policy. In summary, the study underscores the importance of accommodating unobserved heterogeneity in the modeling of commute stop-making decisions.

As indicated earlier in the paper, a limitation of the current model is that it confines attention to the number of commute stop-making. It does not model the many attributes of the evening commute stops (such as stop purpose, stop duration, stop location, etc.) and does not consider commute stop-making within the larger context of overall individual activity-travel patterns. An important area for further research is to pursue such a modeling effort, while at the same time accommodating unobserved heterogeneity across individuals and intra-individual day-to-day variations in activity behavior.

\section{Acknowledgements}

This research was supported by National Science Foundation grants DMS 9208758 and DMS 9313013 to the National Institute of Statistical Sciences (NISS). Thanks are also due to Mr. Charles Purvis of the Metropolitan Planning Commission in Oakland, California for providing the data and for clarifying data issues. Three anonymous referees provided valuable suggestions for improvement on an earlier version of the paper. 


\section{References}

Adiv, A. (1983) The structure of the work-trip based on analysis of trip diaries in the San Francisco bay area. In S. Carpenter \& P. Jones (eds.), Recent Advances in Travel Demand Analysis, 335-349, Gower, Aldershot, England.

Agyemang-Duah, K. and F.L. Hall (1997) Spatial transferability of an ordered response model of trip generation. Transportation Research, 31A, 389-402.

Agyemang-Duah, K., Anderson, W.P., and F.L. Hall (1995) Trip generation for shopping travel. Transportation Research Record, 1493, 12-20.

Bianco, M.J. and C. Lawson (1996) Trip chaining, childcare and personal safety: critical issues in women's travel behavior. Paper presented at the Second National Conference on Women's Travel Issues, Baltimore, Maryland, October.

Bhat, C.R. (1997) Work travel mode choice and number of nonwork commute stops. Transportation Research, 31B, 41-54.

Bhat, C.R. (1998a) Accommodating variations in responsiveness to level-of-service measures in travel mode choice modeling. Transportation Research, 32A, 495-507.

Bhat, C.R. (1998b) Modeling the Commute Activity-Travel Pattern of Workers: Formulation and Empirical Analysis. Working Paper, Department of Civil Engineering, University of Texas at Austin.

Bhat, C.R. and Sujit Singh (1998) A Comprehensive Daily Activity-Travel Generation Model System for Workers. Transportation Research, in press.

Chamberlain, G. (1980) Analysis of Covariance with Qualitative Data. Review of Economic Studies, 47, 225-238.

Davidson, D. (1991) Impact of suburban employee trip chaining on transportation demand management. Transportation Research Record, 1321, 82-89.

Diggle, P.J., K-Y. Liang and S.L. Zeger (1994) Analysis of Longitudinal Data. Clarendon Press, Oxford.

Greene, W.H. (1997) Econometric Analysis. Prentice Hall, New Jersey.

Hamed, M.M and F.L. Mannering (1993) Modeling travelers' postwork activity involvement: toward a new methodology. Transportation Science, 27, 4, 381-394.

Hajivassiliou, V.A. and P.A. Ruud (1994) Classical estimation methods for LDV models using simulation. In Engle, R.F. and D.L. McFadden (eds.) Handbook of Econometrics, 4, 2383-2441.

Hsiao, C. (1986) Analysis of Panel Data. Cambridge University Press, Cambridge.

Jones, P. M., F.S. Koppelman, and J.P. Orfeuil (1993) Activity analysis: state of the art and future directions. In Developments in Dynamic and Activity-Based Approaches to Travel Analysis, 34-55, Gower, Aldershot, England.

Jou, R-C and H.S. Mahmassani (1997) Comparative analysis of day-to-day trip chaining behavior of urban commuters in two cities. Transportation Research Record, in press. 
Kitamura, R., T. Yamamoto, S. Fujii and S. Sampath (1996). A discrete-continuous analysis of time allocation to two types of discretionary activities which accounts for unobserved heterogeneity. In J.B. Lesort (editor) Transportation and Traffic Theory, 431-453, Elsevier, Oxford.

Kondo, K. and R. Kitamura (1987) Time-space constraints and the formation of trip chains. Regional Science and Urban Economics, 17, 49-65.

Lee, L.-F. (1992) On the efficiency of methods of simulated moments and maximum simulated likelihood estimation of discrete response models. Econometric Theory, 8(4), 518-552.

Lockwood, P.B. and M.J. Demetsky (1994) Nonwork travel - a study of changing behavior. Presented at the 73rd Annual Meeting of the Transportation Research Board, Washington, D.C., January.

Maddala, G.S. (1983) Limited Dependent and Qualitative Variables in Econometrics. Cambridge University Press, Cambridge.

McKelvey, R.D. and W. Zavoina (1975). A statistical model for the analysis of ordinal level dependent variables. Journal of Mathematical Sociology, 4, 103-120.

McMillen, D.P. (1995). Spatial effects in probit models: a monte carlo investigation. In L. Anselin and R.J.G.M. Florax (editors) New Directions in Spatial Econometrics, Springer-Verlag, New York.

Mensah, J. (1995) Journey to work and job search characteristics of the urban poor: a gender analysis of a survey data from Edmonton, Alberta. Transportation, 22, 1-19.

Nishii, K., K. Kondo and R. Kitamura (1988) Empirical analysis of trip chaining behavior. Transportation Research Record, 1203, 48-59.

Purvis, C.L. (1994) Changes in regional travel characteristics and travel time budgets in the San Francisco bay area: 1960-1990. Presented at the 73rd Annual Meeting of the Transportation Research Board, Washington, D.C., January.

Purvis, C.L. (1996) Estimation of Home-Based Work Mode Choice Models. Technical Memorandum in San Francisco Bay Area 1990 Travel Demand Model Development Project, Planning Section, Bay Area Metropolitan Transportation Commission.

Revelt, D. and K. Train (1997) Mixed logit with repeated choices: households' choices of appliance efficiency level. Review of Economics and Statistics, in press.

Strathman, J.G., K.J. Dueker and J.S. Davis (1994). Effects of household structure and selected travel characteristics on trip chaining. Transportation, 21, 23-45.

Swait, J. and W. Adamowicz (1996) The effect of choice environment and task demands on consumer behavior: discriminating between contribution and confusion. Working paper, Department of Rural Economy, University of Alberta, Canada.

Train, K. (1997) Recreation demand models with taste differences over people. Land Economics, in press.

White, E.H. and Company, Inc. (1991) 1990 bay area travel survey: final report. The Metropolitan Transportation Commission, Oakland, CA, submitted for publication. 